\title{
Anaesthetic Options in Chronic Inflammatory Demyelinating Polyneuropathy
}

\section{Rivas BH*, Romero RP and Sánchez JLA}

Department of Anesthesiology, Son Llàtzer Hospital, Palma of Mallorca, Spain

\begin{abstract}
Chronic Inflammatory Demyelinating Polyneuropathy (CIDP) is an acquired inflammatory disorder of unknown etiology affecting the peripheral nervous system. The disease can be chronically progressive or follow a pattern of relapses and remissions. Motor involvement is usually greater than sensory and causes symmetrical, proximal limb weakness. CIDP is not a common disease in our field. Ignorance in the anesthetic management of these patients creates uncertainty. There are not any guidelines for the specific anaesthetic management of these patients. The possibility of a complications after an anesthetic action has motivated us to share our experience and conclusions after a brief review. The patient's permission was obtained to publish this case report.
\end{abstract}

Keywords: Chronic inflammatory demyelinating polyneuropathy (CIDP); Peripheral nervous system; Spinal anaesthesia

\section{Case Report}

A 65 year old male was scheduled for surgery for a left intertrochanteric femur fracture. His medical history included insulin-dependent diabetes mellitus, a two-year history of diabetic polyneuropathy, CIDP, Hashimoto's thyroiditis, fatty liver disease and a simple hepatic cyst.

In August 2011, a neurology study was undertaken due to symptoms of latent weakness, paraparesis and muscle atrophy in the lower limbs. In February 2012, he was admitted with a ten-day history of exacerbation of his neurological symptoms, with cancer, autoimmune disease and infection all being ruled out as possible secondary causes. He was finally diagnosed with CIDP according to the Cornblath criteria, although he only met criteria for possible CIDP according to European Federation of Neurological Societies/Peripheral Nerve Society guidelines (2011). He was treated with intravenous methylprednisolone for five days and intravenous immunoglobulins for four days, with improvement evident.

In the preoperative anaesthetic assessment, he referred that his neurological symptoms had not worsened since diagnosis. There were no signs of any upper-limb motor deficit. At a sensory level, he had glove and stocking hypoesthesia associated with his diabetic polyneuropathy. According to the Medical Research Council Scale (Table 1), muscle power in his lower limbs was as follows: hip flexors (right/left): 4/4-; quadriceps 4/3; hamstrings 4/4-; dorsiflexors 4/5. Knee and ankle jerk reflexes were absent. No abnormalities involving the cranial nerves were found.

Once in the operating theatre, the patient underwent the following: standard monitoring (Datex Ohmeda ${ }^{\oplus}$ Monitor), cannulation with an $18 \mathrm{G}$ peripheral line $\left(\right.$ Braun $\left.^{\oplus}\right)$, oxygenation via nasal cannulae at $2 \mathrm{lpm}$ and sedation with $2 \mathrm{mg}$ of midazolam. Spinal anaesthesia achieving subarachnoid block was performed in the sitting position with a G25 pencil-point needle (Braun ${ }^{\star}$ ) and $10 \mathrm{mg}$ of $0.5 \%$ hyperbaric bupivacaine $\left(\right.$ Braun $\left.^{\circledR}\right)$ administered through the L3/L4 space. The "pinprick test" showed that sensory block had reached T10 by ten minutes after the puncture. Reduction and insertion of a proximal femoral nail antirotation was then carried out. The patient's haemodynamic and respiratory status remained stable throughout the one hundred minutes of surgery and while in recovery. Two hundred minutes after administration of the spinal anaesthesia, he had recovered full flexion of knees and feet. Following sensory-motor recovery, he returned to his baseline status. During his time in hospital, he suffered no changes or exacerbation of his usual symptoms.

\section{Discussion}

CIDP is an acquired demyelinating disorder affecting the peripheral nervous system which can be chronically progressive or follow a pattern of relapses and remissions [1].

Although the aetiology is unknown, one hypothesis is that this polyneuropathy is immunologically based and involves both humoral and cellular components of the immune system. The prevalence of CIDP is $0.8-8.9$ per 100,000 [1], it represents $20 \%$ of undiagnosed neuropathies and it can affect all ages.

It generally starts gradually over months, but there are cases of acute onset indistinguishable from Guillain-Barre syndrome. The disease course tends to be slowly progressive, but a variant with relapses and remissions has also been described in younger patients. Symptoms are both motor and sensory, although motor involvement is usually greater. Limb weakness is almost always symmetrical, predominantly affecting the proximal muscles of the arms and legs. An overall decrease or absence of deep tendon reflexes is found in most cases. Patients can also develop autonomic dysfunction and a few have cranial nerve involvement. Hypertrophy of the nerve roots can cause dysaesthesia, cauda equina syndrome and symptoms of lumbar stenosis, which sometimes require surgery [1].

Suspected diagnosis is based on the symptoms and their progressive development over more than two months [2]. Different studies are required to confirm the diagnosis: electrophysiological examination will show peripheral demyelination (conduction bock, slowing of conduction velocity - prolonged distal latencies or delay/disappearance of F-waves, and distal, temporal dispersion of motor action potential);

*Corresponding author: Beatriz Hernández Rivas, Department of Anesthesiology Institution: Deparment of Anesthesiology, Son Llàtzer Hospital, Carretera de Manacor, 07198 Palma de Mallorca, Spain,Tel: 00-34-871-20-2133; Fax: 00-34871-20-2359; E-mail: bea_heriv87@hotmail.es

Received September 25, 2014; Accepted November 07, 2014; Published November 09, 2014

Citation: Rivas BH, Romero RP, Sánchez JLA (2014) Anaesthetic Options in Chronic Inflammatory Demyelinating Polyneuropathy. J Clin Case Rep 4: 457. doi:10.4172/2165-7920.1000457

Copyright: ( 2014 Rivas BH, et al. This is an open-access article distributed under the terms of the Creative Commons Attribution License, which permits unrestricted use, distribution, and reproduction in any medium, provided the original author and source are credited. 


\begin{tabular}{|c|l|}
\hline & Modified Mrc Muscle Power Scale \\
\hline $\mathbf{0 / 5}$ & Total absence of voluntary contraction \\
\hline $\mathbf{1 / 5}$ & Contraction is seen or felt but no movement \\
\hline $\mathbf{2 / 5}$ & Movement occurs but cannot move against gravity \\
\hline $\mathbf{3 - 1 5}$ & Active movement against gravity but cannot achieve full range of movement of the segment examined \\
\hline $\mathbf{3 + / 5}$ & Active movement against gravity and some resistance exerted but quickly cedes \\
\hline $\mathbf{4 - / 5}$ & Active movement against gravity and minimal resistance exerted by examiner \\
\hline $\mathbf{4 / 5}$ & Active movement against gravity and moderate resistance exerted by examiner \\
\hline $\mathbf{4 + / 5}$ & The muscle is slightly weak \\
\hline $\mathbf{5 - 1 5}$ & The muscle is somewhat weak \\
\hline $\mathbf{5 / 5}$ & Normal power \\
\hline
\end{tabular}

Table 1: Modified medical research council scale for muscle strength*. UK Medical Research Council [11].

\begin{tabular}{|l|l|l|l|}
\hline Author & Anaesthesia & Surgery & Observations/Outcome \\
\hline Hara et al. [6] & General anaesthetic & Gastrectomy and colectomy & Prolongation of the effect of the rocuronium. Normal recovery \\
\hline Schabel et al. [7] & Subarachnoid anaesth. & Caesarean (36 weeks) ${ }^{\star *}$ & Improved symptoms \\
\hline Galan et al. [8] & Femoral block + sciatic block & Bimalleolar fracture & Anaesthetic effect and normal recovery. Stronger electrical currents for sciatic stimulation \\
\hline Gupta et al. [9] & Subarachnoid anaesth. & Fractured neck of femur & Anaesthetic effect and normal recovery \\
\hline Richter et al. [10] & Subarachnoid anaesth. & Caesarean (38 weeks) ${ }^{\star *}$ & Dorsiflexion recovery $>15$ h. Over 24 h before recovered pre-anaesthetic status \\
\hline
\end{tabular}

**Both caesareans were performed due to worsening of CIDP symptoms and the contraindication for specific therapy in pregnant women.

Table 2: Table summarizing the anesthetic technique, surgery and results of published cases.

cerebrospinal fluid analysis reveals an increase in proteins without pleocytosis; nerve biopsy shows evidence of endoneurial edema, onion bulb formation and endoneurial mononuclear cell infiltration. There is some debate about the diagnostic utility of performing a biopsy on the sural nerve [2].

In terms of treatment, in mild cases of CIDP, a wait-and-see approach may be adopted. In severe or rapidly progressive cases, the treatment is based on IV immunoglobulins, plasmapheresis or corticosteroids. Patients may also benefit from the addition of immunosuppressants. Up to one third of patients with this disease do not respond adequately to the initial treatment chosen and have to switch to a different one. In $75 \%$ of cases treated, reasonable functional recovery is achieved after years of treatment [1].

The choice of anaesthetic technique for patients with CIDP is complicated. We were unable to find any recommendations on the anaesthetic management of these patients. Through a literature review, we found five articles [3-11] on patients with CIDP who underwent surgery using some type of anaesthetic technique (Table 2).

The lack of literature on the anaesthetic management of CIDP patients forced us to review similar neurological conditions such as Guillain-Barre Syndrome (GBS) and Charcott-Marie-Tooth type 1 (CMT1). These three diseases are all peripheral polyneuropathies with motor involvement and myelin degeneration.

GBS is an acute autoimmune polyradiculoneuropathy. The annual incidence is $1-4$ cases per 100,000 people It is a rapidly progressing disorder involving motor paralysis and areflexia, with or without sensory loss. The most typical pattern is of ascending paralysis.

Depolarising muscle relaxants (DMR) are contraindicated in GBS due to the high risk of hyperkalaemia and malignant hyperthermia. The reason behind this may be an overexpression of cholinergic receptors in the acute phase of the disorder that trigger a massive outflow of potassium. Cases of cardiac arrest have been reported in induction of anaesthesia with succinylcholine. The use of non-depolarising muscle relaxants (NDMR) in GBS can lead to longer duration of the neuromuscular blockade or to resistance, depending on the stage of the disease; if used, muscle relaxation must be monitored. Use of inhaled anaesthetic agents has no clinical implications related to the pathophysiology of GBS. Patients with GBS do, however, seem to have greater sensitivity to Local Anaesthetics (LA) and this may cause greater spread of the sympathetic block than expected, with exacerbation of the hemodynamic effects. Should the patient develop hypotension, direct-acting sympathomimetic agents should be used, as the response to the indirect-acting drugs is unpredictable [1]. Compared to general anaesthesia, neuraxial analgesia does not seem to cause exacerbation of the disease or trigger new crises in cured patients. Cautious administration of LA to establish the desired level of blockade is recommended.

Charcot-Marie-Tooth 1 (CMT1) is the most common form of hereditary neuropathy. The prevalence is $10-30$ cases per 100,000 people. Symptoms begin between late childhood and early adulthood with weakness in the lower legs. Anaesthetic considerations described in CMT1 are similar to other neuropathy like GBS. Although studies have shown variability in the effect of NDMR (ranging from increased, to decreased, to normal) [1] their use is permitted. Both propofol and fentanyl are considered safe, despite there are opioids which could lead to increased respiratory depression. Debate surrounds the use of LA due to the direct toxic effects of these drugs on the demyelinated nerve. There are reports of neuraxial block in which no abnormal effects such as nerve injury or exacerbation of CMT were observed.

With our patient, we assessed both his disease and the surgical intervention he required. In patients with CIDP, the phase of the disease, the length of time they have remained stable and the type of surgery can all be decisive. Prior to the intervention, we must rule out any signs of the patient being in an acute phase of the disease with a thorough history and physical examination. The assessment of muscle strength on admission allow us to rule out any change from his baseline status after surgery. If opting for a general anaesthetic, both DMR and NDMR must be avoided. In our case the risk of possible hypersensitivity to $\mathrm{DMR}^{3}$ was what led us to opt for a subarachnoid block.

If the patient has to be intubated, NDMR are the drugs of choice and monitoring of the relaxation is mandatory. There is only one reported case of general anesthesia in patient with CIDP by Hara [6] et al. they observed a prolonged effect of rocuronium. 
Citation: Rivas BH, Romero RP, Sánchez JLA (2014) Anaesthetic Options in Chronic Inflammatory Demyelinating Polyneuropathy. J Clin Case Rep 4: 457. doi:10.4172/2165-7920.1000457

Neuraxial techniques appear to be safe, bearing in mind the possibility of a larger block level than expected. With peripheral nerve block, the potential for direct nerve injury has to be considered. However, the publication of a case which transpired without incident encourages us to view it as a potentially safe option.

\section{Conclusions}

The choice of anaesthetic technique should be made on an individual basis for each patient. We can conclude that there is no completely safe technique and that any anaesthetic or surgical procedure could trigger an exacerbation in a neurological disease such as CIDP. Leaning towards one or another will depend on the assessment of the riskbenefit ratio in each case.

Because of the limitation of our study based on an observational case and the absence of guidelines on managing these patients, do not allow us to make any recommendations based on science. So we encourage other colleagues to describe their experiences with these patients in order to improve our practices.

\section{References}

1. Hauser SL, Amato AA (2012) Sindrome de Guillain-Barré y otras neuropatías mediadas por mecanismos inmunitarios. Harrison principios de medicina interna, (18thedn). Mexico: McGraw-Hill Ryerson.
2. http://www.uptodate.com/

3. Butterworth JF, Mackey DV, Wasnick JD (2013) Morgan \& Mikhail's Clinical Anesthesiology. (5th edn), New York, NY: Lange Medical Books/ McGraw-Hill.

4. Brooks H, Christian AS, May AE (2000) Pregnancy, anaesthesia and Guillain Barré syndrome. Anaesthesia 55: 894-898.

5. Pogson D, Telfer J, Wimbush S (2000) Prolonged vecuronium neuromuscular blockade associated with Charcot Marie Tooth neuropathy. $\mathrm{Br} \mathrm{J}$ Anaesth 85 914-917.

6. Hara K, Minami K, Takamoto K, Shiraishi M, Sata T (2000) The prolonged effect of a muscle relaxant in a patient with chronic inflammatory demyelinating polyradiculoneuropathy. Anesth Analg 90: 224-226

7. Schabel JE (2001) Subarachnoid block for a patient with progressive chronic inflammatory demyelinating polyneuropathy. Anesth Analg 93:1304-1306.

8. http://zl.elsevier.es/es/revista/revista-espanola-anestesiologiareanimacion-344/linkresolver/bloqueo-nervioso-periferico-guiadopor-90267293

9. Gupta B, Agrawal P, D'souza N, Sawhney C (2011) Anaesthetic management and implications of a case of chronic inflammatory demyelinating polyneuropathy. Indian J Anaesth 55: 277-279.

10. Richter T, Langer KA, Koch T (2012) Spinal anesthesia for cesarean section in a patient with chronic inflammatory demyelinating polyradiculoneuropathy. $J$ Anesth 26: 280-282.

11. UK Medical Research Council (1976) Aids to the Investigation of Peripheral Nerve Injuries. N 45: 6-7. 